\title{
Prognostic Factors for the Development of Biochemical Recurrence after Radical Prostatectomy
}

\author{
Ahmed F. Kotb and Ahmed A. Elabbady \\ Urology Department, Faculty of Medicine, Alexandria University, Alexandria, Egypt \\ Correspondence should be addressed to Ahmed A. Elabbady, a_elabbady@hotmail.com \\ Received 28 February 2011; Revised 8 April 2011; Accepted 19 April 2011 \\ Academic Editor: Kenneth A. Iczkowski
}

Copyright (C) 2011 A. F. Kotb and A. A. Elabbady. This is an open access article distributed under the Creative Commons Attribution License, which permits unrestricted use, distribution, and reproduction in any medium, provided the original work is properly cited.

\begin{abstract}
Prostate cancer is one of the most common cancers in Western countries and is associated with a considerable risk of mortality. Biochemical recurrence following radical prostatectomy is a relatively common finding, affecting approximately $25 \%$ of cases. The aim of our paper was to identify factors that can predict the occurrence of biochemical recurrence, so the patient can be properly counselled pre- and postoperatively. Medline review of the literatures was done followed by a group discussion on the chosen publications and their valuable influence. Preoperative serum total PSA and clinical stage, together with prostatectomy Gleason grade, tumour volume, and perineural and vascular invasions, were the most important variables found to influence outcome.
\end{abstract}

\section{Introduction}

In Canada, it is estimated that 1 in 7 men will develop prostate cancer (Pca) during their lifetime, and 1 of 27 will die of it (a ratio of 1 death per 4 diagnosed cases) [1]. In 2009, approximately 192.000 men from the USA were diagnosed with prostate cancer, with an estimate of 27.000 of those men dying from it [2].

The Food and Drug Administration (FDA) approved PSA testing in 1986 to monitor men with prostate cancer. Since then, PSA was widely used both to screen prostate cancer and to monitor treatment response and recurrence. This resulted in early diagnosis of cases with organ localized disease amenable to definitive treatment and in early diagnosis of disease recurrence following surgery with early treatment before widespread dissemination.

The ideal therapy for clinically localized prostate cancer is still controversial. Although radical prostatectomy (RP) is a valid option, the rate of biochemical recurrence following open or laparoscopic prostatectomy is estimated to be 15$40 \%[3,4]$.

As PSA may require up to 8 weeks to be cleared from the circulation [5], biochemical recurrence cannot be diagnosed before that time frame, following radical prostatectomy. The American Urological Association and EAU defined biochemical recurrence (BCR) following $\mathrm{RP}$ as an initial serum PSA of greater than or equal to $0.2 \mathrm{ng} / \mathrm{mL}$, with a second confirmatory level of PSA greater than $0.2 \mathrm{ng} / \mathrm{mL}$ $[6,7]$. Freedland et al. attempted to define the lowest PSA cut point associated with PSA progression within 1 year, along with $100 \% 3$-year risk of progression [8]. They concluded that a $0.2 \mathrm{ng} / \mathrm{mL}$ represented the most conservative cut point to define BCR, after RP. Stephenson et al. subsequently examined the correlation between 10 definitions of biochemical recurrence with metastatic progression in a clinical cohort [9]. They proposed that biochemical recurrence should be defined as a PSA $>0.4 \mathrm{ng} / \mathrm{mL}$ with a confirmatory rise. Hattab et al. defined biochemical recurrence as two consecutive serum PSA measurements $>0.1 \mathrm{ng} / \mathrm{mL}$ [10]. Regardless of which definition may be better in defining BCR, the aim is to identify the perioperative factors associated with a high risk of BCR, so that patients proved to be at a higher risk of BCR can be followed more closely postoperatively and may be advised for early adjuvant treatment, after RP. Cronin et al. studied 5473 patients, who underwent RP from 1985 to 2007 [11]. An analysis of perioperative clinical and pathological parameters was done with 12 definitions of BCR. They observed that the relative risks and hazard ratios were fairly consistent between the 12 definitions examined, and the statistical inference on established prognostic factors was not 
impacted by the definition of BCR. So research groups using different definitions for BCR seem to reach similar conclusions regarding prognostic factors.

We discuss some factors we believe to be of important influence on the progression of prostate cancer, following its definitive management.

\section{Preoperative Factors}

2.1. Clinical Stage. The 2002 American Joint Committee on cancer TNM clinical staging system for prostate cancer substratified localized disease as T1 and T2. According to this committee, $\mathrm{T} 1$ represents disease with no abnormality on DRE and T2 represents a DRE palpable disease which is further subclassified into T2a, b, and c according to the extent of prostatic lobes affected [12]. D'Amico et al. developed a risk stratification system to predict BCR, after RP [13]. Both T1c and T2a were considered low-risk groups, whereas $\mathrm{T} 2 \mathrm{~b}$ and T2c were classified as intermediate- and high-risk groups, respectively. More recently, Billis et al. described different clinicopathological characteristics between patients with clinical stage T1c versus stages $\mathrm{T} 2 \mathrm{a}$ and $\mathrm{T} 2 \mathrm{~b}$ [14]. Freedland et al. also showed no difference in BCR rate between patients with clinical stage T2a and T2b, with excellent 10-year progression-free survival in both groups [15].

Cooperberg et al. reported the San Francisco experience in prostate cancer risk assessment [16]. They demonstrated that clinical stage was not a predictive factor for biochemical recurrence in their model. Armatys et al. confirmed that cases with cT2 had worse pathological outcomes than cT1c [17]. However, no statistical difference between both groups could be found regarding BCR rate. Reese et al. reported that clinical stage in 4899 men who underwent RP for localized Pca was predictive for BCR on univariate, rather than multivariate, studies [18].

Stephenson et al. included clinical stage in their nomogram to predict BCR following RP [19]. Hashimoto et al. showed that clinical stage $\geq \mathrm{T} 2 \mathrm{a}$ is highly significant in predicting positive surgical margin (SM) following RP [20]. In their cohort, positive SM was significantly predicting a higher rate of BCR, although they did not directly examine the association between the clinical stage and the BCR. In a study on Japanese populations, Egawa et al. found that clinical stage contributed significantly in the prediction of biochemical recurrence, in their cohort of patients [21].

We confirm DRE to be an integral part in the routine urological examination, for men above 55 years of age. Suspicious findings on DRE can predict worse outcomes following definitive management, compared to cases where diagnosis was based on high serum PSA with normal DRE findings.

2.2. Preoperative Serum PSA and PSA Density (PSAd). PSA and PSAd are factors predicting BCR in most of the available nomograms and studies $[16,18,22,23]$. Radwan et al. reported that PSAd, whether measured using U/S or the true prostate weight, was highly predicting for $\mathrm{BCR}$, more than
PSA [23]. Freedland et al. [24] initially reported that PSAd was a strong predictor for pathological adverse effects and BCR following RP and should be integrated in the risk stratification for Pca. In a more recent study on a larger cohort of patients, Freedland et al. found that preoperative PSAd relative to PSA provides little improvement for predicting BCR following RP [25]. Brassell et al. correlated PSA and PSAd measured using both ultrasound and the true prostate volume [26]. They reported that PSA was significantly better than PSAd in predicting BCR.

From our experience, we believe that PSAd has no added role over PSA alone as a prognostic factor for disease progression in intermediate- and high-risk groups (PSA $>10 \mathrm{ng} / \mathrm{mL}$ ). On the other hand, in low-risk groups, with serum PSA $<10 \mathrm{ng} / \mathrm{mL}$, PSAd is pivotal in predicting patient outcome.

\section{Pathological Factors}

3.1. Gleason Grade. Gleason grade is the most widely accepted grading system for prostatic adenocarcinoma. Given the inherent sampling error of diagnostic needle biopsy and the multifocal nature of this tumor, discrepancy between Gleason score (GS) of needle biopsy and RP specimen was a common finding in the literature. King summarized 11 published series that covered over 2600 patients, in which an accurate match of grading was seen in an average of only $42 \%$ of the cases [27]. We thus believe that Gleason grade obtained from needle biopsy has a limited role in the decision making for patient management, compared to the RP Gleason grading.

Gleason score 7 should not be considered in our opinion as a single disease entity, and whenever the urologist is encountered with GS 7, decision on patient management should be done based on its primary Gleason pattern. Lau et al. studied 263 men with grade 7 RP specimens [28]. They noted that patients with primary Gleason grade 4 had a significantly higher rate of progression than patients with primary Gleason grade 3 (46\% versus 33\%). Sakr et al. performed the same study on 534 men, followed for a mean of 34.6 months [29]. They showed that primary grade 4 was associated with a higher rate of BCR, more than primary grade 3 ( $23 \%$ versus $11 \%$, at 2 -year followup).

Some studies considered the percentage of high Gleason pattern as a factor predicting a higher rate of BCR [30, 31]. On the other hand, Chan et al. reported that high Gleason pattern is not likely to be reproducible [32]. It was often difficult and time consuming and results in a prognostic effect only at its extremes (greater than $70 \%$ or less than $20 \%$ with pattern 4/5).

In our institution, we do not consider the percentage of high Gleason pattern. Nevertheless, we follow the current principals of considering cases with GS $\leq 6$ as low risk, compared to cases with GS $>6$ as high risk.

3.2. Tumour Volume (TV). Data about the effect of tumour volume on BCR are contradictory in the literature. Some studies could not find a correlation between tumor volume 
(TV) and BCR [33, 34], whereas others considered tumour volume a high predictor factor for cancer progression [3539].

Fukuhara et al. used the maximum tumour diameter (MTD) as an estimate for tumour volume, to correlate with BCR [40]. They noted that, although both PSA and MTD significantly correlated with BCR on univariate analysis, only MTD independently predicted BCR on multivariate analysis. These findings were in agreement with those of Chung et al. [41].

In our institution, we prefer measuring the maximal tumour diameter rather than the tumour volume. No data is currently available for us to judge its direct effect on the rate of BCR. We believe it to be an important variable to be included in our further studies.

3.3. Surgical Margin. Blute et al. reported a positive margin rate of $39 \%$ in more than 2500 patients from the Mayo series, and the BFS at 5 years was $67 \%$ and $84 \%$ in patients with positive and negative SM, respectively [42]. Similarly, Hashimoto et al. reported 5-year BFS rate of $62.6 \%$ and $81.7 \%$ for patients with positive and negative SM, respectively [43].

Sæther et al. failed to show SM positivity to be an independent risk factor for BCR although they could show that it had a strong trend to do so $(P=.06)$ [44]

Lake et al. studied the disease-free survival of patients with negative surgical margin versus those with either focal (FPM) or extensive positive surgical margin (EPM) [45]. In their study, the 10-year DFS was $90 \%, 76 \%$, and $53 \%$ for those with negative SM, FPM, and EPM, respectively, and that achieved statistical significance. Ochiai et al. could also show that patients with positive SM longer than $3 \mathrm{~mm}$ had much lower outcomes than those with shorter positive SM [46].

In our experience, a positive surgical margin is not a factor toward a significantly higher rate of BCR, although we consider it an indication for adjuvant radiotherapy.

3.4. Perineural Invasion (PNI). A recent study from Italy reported that $65.7 \%$ of their prostatectomy specimens that were associated with higher Gleason grades had PNI, but not BCR [47]. Merrilees et al. detected PNI in $90 \%$ of their 105 prostatectomy specimens [48]. They also concluded that PNI was not a significant predictor for the occurrence of BCR. Miyake et al. found PNI to be of significance to predict BCR on univariate analysis but not multivariate analysis [49]. Endrizzi and Seay found PNI in their 131 patients to be a significant factor predicting BCR, with a sensitivity of $82 \%$ and a specificity of $57 \%$ [50].

Beard et al. studied the association of PNI and the relapse rate following external beam radiation [51]. They reported a significant association of PNI with higher Gleason score and a higher rate of BCR on univariate analysis. On multivariate analysis, PNI was not an independent factor predicting BCR. O'Malley et al. studied 78 patients with PNI detected by prostatic biopsy with other 78 patients with absent PNI [52]. They reported no significant effect of PNI on the rate of BCR following RP, when adjusted to GS, PSA, and pathological stage.

3.5. Vascular Invasion (VI). Van den Ouden et al. reported vascular invasion (VI) in $12 \%$ of their patients who underwent prostatectomy [53]. VI was a significant factor in predicting BCR on univariate and multivariate analysis. De la Taille et al. found VI in $12.4 \%$ of their cases and confirmed VI to be an independent significant factor predicting BCR [54]. Ferrari et al. detected VI in $18 \%$ of their patients and confirmed that VI was associated with worst pathological outcomes and was an independent factor predicting BCR on long-term followup [55]. The College of American Pathologists recommended VI to be assessed routinely in radical prostatectomy specimens [56].

3.6. Neuroendocrinal Differentiation. Quek et al. studied the correlation between neuroendocrinal (NE) expression, using $\mathrm{CgA}$, in malignant and benign acini and recurrences [57]. They detected statistically significant association of the number of NE cells in only malignant acini and clinical recurrence. Weinstein et al. also showed that the number of malignant NE cells is of prognostic significance for patients undergoing radical prostatectomy [58]. In a multivariate analysis, May et al. [59] detected neuroendocrinal differentiation to be the second most significant predictor for biochemical progression. On the other hand, other authors did not find a significant association between NE differentiation and disease progression or biochemical failure $[60,61]$.

Although there are no solid data on the influence of neuroendocrinal differentiation on BCR and although it is currently not performed as a routine staining in our institution, we recommend staining for NE cells within the prostate as this may serve to predict biochemical recurrence as proposed by some. It may also point toward considering early chemotherapy in relapsing cases with higher degree of $\mathrm{NE}$ differentiation.

\section{Conclusion}

There is wide consensus that biochemical recurrence occurs in a large percentage of patients following successful radical prostatectomy. No single factor can be considered toward the prediction of recurrence. Preoperative PSA, clinical stage, prostatectomy Gleason grade, tumour volume, perineural invasion, and vascular invasion are the most important clinical and pathological parameters for assessing after radical prostatectomy. Neuroendocrinal differentiation may be an important pathological criterion to look for in a prostatectomy specimen.

\section{References}

[1] Y. Fradet, L. Klotz, J. Trachtenberg, and A. Zlotta, "The burden of prostate cancer in Canada," Journal of the Canadian Urological Association, vol. 3, no. 3, supplement 2, pp. S102S108, 2009. 
[2] A. Jemal, R. Siegel, E. Ward, Y. Hao, J. Xu, and M. J. Thun, "Cancer statistics, 2009," CA: Cancer Journal for Clinicians, vol. 59, no. 4, pp. 225-249, 2009.

[3] P. C. Walsh, A. W. Partin, and J. I. Epstein, "Cancer control and quality of life following anatomical radical retropubic prostatectomy: results at 10 years," Journal of Urology, vol. 152, no. 5, pp. 1831-1836, 1994.

[4] H. A. Frazier, J. E. Robertson, P. A. Humphrey, and D. F. Paulson, "Is prostate specific antigen of clinical importance in evaluating outcome after radical prostatectomy," Journal of Urology, vol. 149, no. 3, pp. 516-518, 1993.

[5] J. E. Oesterling, "Prostate specific antigen: a critical assessment of the most useful tumor marker for adenocarcinoma of the prostate," Journal of Urology, vol. 145, no. 5, pp. 907-923, 1991.

[6] M. S. Cookson, G. Aus, A. L. Burnett et al., "Variation in the definition of biochemical recurrence in patients treated for localized prostate cancer: the American Urological Association Prostate Guidelines for Localized Prostate Cancer Update Panel report and recommendations for a standard in the reporting of surgical outcomes," Journal of Urology, vol. 177, no. 2, pp. 540-545, 2007.

[7] G. Aus, C. C. Abbou, M. Bolla et al., "EAU guidelines on prostate cancer," European Urology, vol. 48, no. 4, pp. 546-551, 2005.

[8] S. J. Freedland, M. E. Sutter, F. Dorey, and W. J. Aronson, "Defining the ideal cutpoint for determining PSA recurrence after radical prostatectomy," Urology, vol. 61, no. 2, pp. 365$369,2003$.

[9] A. J. Stephenson, M. W. Kattan, J. A. Eastham et al., "Defining biochemical recurrence of prostate cancer after radical prostatectomy: a proposal for a standardized definition," Journal of Clinical Oncology, vol. 24, no. 24, pp. 3973-3978, 2006.

[10] E. M. Hattab, M. O. Koch, J. N. Eble, H. Lin, and L. Cheng, "Tertiary Gleason pattern 5 is a powerful predictor of biochemical relapse in patients with Gleason scores 7 prostatic adenocarcinoma," Journal of Urology, vol. 175, no. 5, pp. 16951699, 2006.

[11] A. M. Cronin, G. Godoy, and A. J. Vickers, "Definition of biochemical recurrence after radical prostatectomy does not substantially impact prognostic factor estimates," Journal of Urology, vol. 183, no. 3, pp. 984-989, 2010.

[12] F. L. Greene, AJCC Cancer Staging Manual, Springer, New York, NY, USA, 6th edition, 2002.

[13] A. V. D'Amico, R. Whittington, S. Bruce Malkowicz et al., "Biochemical outcome after radical prostatectomy, external beam radiation therapy, or interstitial radiation therapy for clinically localized prostate cancer," Journal of the American Medical Association, vol. 280, no. 11, pp. 969-974, 1998.

[14] A. Billis, L. A. Magna, I. C. Watanabe, M. V. Costa, G. H. Telles, and U. Ferreira, "Are prostate carcinoma clinical stages T1c and T2 similar?" International Brazilian Journal of Urology, vol. 32, no. 2, pp. 165-171, 2006.

[15] S. J. Freedland, A. W. Partin, J. I. Epstein, and P. C. Walsh, "Biochemical failure after radical prostatectomy in men with pathologic organ-confined disease: pT2a versus pT2b," Cancer, vol. 100, no. 8, pp. 1646-1649, 2004.

[16] M. R. Cooperberg, D. J. Pasta, E. P. Elkin et al., "The University of California, San Francisco Cancer of the Prostate Risk Assessment score: a straightforward and reliable preoperative predictor of disease recurrence after radical prostatectomy," Journal of Urology, vol. 173, no. 6, pp. 1938-1942, 2005.

[17] S. A. Armatys, M. O. Koch, R. Bihrle, T. A. Gardner, and L. Cheng, "Is it necessary to separate clinical stage T1c from T2 prostate adenocarcinoma?" BJU International, vol. 96, no. 6, pp. 777-780, 2005.

[18] A. C. Reese, M. R. Cooperberg, and P. R. Carroll, "Minimal impact of clinical stage on prostate cancer prognosis among contemporary patients with clinically localized disease," Journal of Urology, vol. 184, no. 1, pp. 114-119, 2010.

[19] A. J. Stephenson, P. T. Scardino, J. A. Eastham et al., "Preoperative nomogram predicting the 10 -year probability of prostate cancer recurrence after radical prostatectomy," Journal of the National Cancer Institute, vol. 98, no. 10, pp. 715-717, 2006.

[20] K. Hashimoto, N. Masumori, F. Takei et al., "Prognostic value of surgical margin status for biochemical recurrence following radical prostatectomy," Japanese Journal of Clinical Oncology, vol. 38, no. 1, pp. 31-35, 2008.

[21] S. Egawa, K. Suyama, Y. Arai et al., "A study of pretreatment nomograms to predict pathological stage and biochemical recurrence after radical prostatectomy for clinically resectable prostate cancer in Japanese Men," Japanese Journal of Clinical Oncology, vol. 31, no. 2, pp. 74-81, 2001.

[22] H. Isbarn, M. Wanner, G. Salomon et al., "Long-term data on the survival of patients with prostate cancer treated with radical prostatectomy in the prostate-specific antigen era," $B J U$ International, vol. 106, no. 1, pp. 37-43, 2010.

[23] M. H. Radwan, Y. Yan, J. R. Luly et al., "Prostate-specific antigen density predicts adverse pathology and increased risk of biochemical failure," Urology, vol. 69, no. 6, pp. 1121-1127, 2007.

[24] S. J. Freedland, J. A. Wieder, G. S. Jack, F. Dorey, J. B. Dekernion, and W. J. Aronson, "Improved risk stratification for biochemical recurrence after radical prostatectomy using a novel risk group system based on prostate specific antigen density and biopsy Gleason score," Journal of Urology, vol. 168, no. 1, pp. 110-115, 2002.

[25] S. J. Freedland, C. J. Kane, J. C. Presti et al., "Comparison of preoperative prostate specific antigen density and prostate specific antigen for predicting recurrence after radical prostatectomy: results from the search data base," Journal of Urology, vol. 169, no. 3, pp. 969-973, 2003.

[26] S. A. Brassell, T. C. Kao, L. Sun, and J. W. Moul, "Prostatespecific antigen versus prostate-specific antigen density as predictor of tumor volume, margin status, pathologic stage, and biochemical recurrence of prostate cancer," Urology, vol. 66, no. 6, pp. 1229-1233, 2005.

[27] C. R. King, "Patterns of prostate cancer biopsy grading: trends and clinical implications," International Journal of Cancer, vol. 90, no. 6, pp. 305-311, 2000.

[28] W. K. Lau, M. L. Blute, D. G. Bostwick, A. L. Weaver, T. J. Sebo, and H. Zincke, "Prognostic factors for survival of patients with pathological gleason score 7 prostate cancer: differences in outcome between primary gleason grades 3 and 4," Journal of Urology, vol. 166, no. 5, pp. 1692-1697, 2001.

[29] W. A. Sakr, M. V. Tefilli, D. J. Grignon et al., "Gleason score 7 prostate cancer: a heterogeneous entity? Correlation with pathologic parameters and disease-free survival," Urology, vol. 56, no. 5, pp. 730-734, 2000.

[30] M. Noguchi, T. A. Stamey, J. E. McNeal, and C. M. Yemoto, "Preoperative serum prostate specific antigen does not reflect biochemical failure rates after radical prostatectomy in men with large volume cancers," Journal of Urology, vol. 164, no. 5, pp. 1596-1600, 2000.

[31] L. Cheng, M. O. Koch, B. E. Juliar et al., "The combined percentage of gleason patterns 4 and 5 is the best predictor 
of cancer progression after radical prostatectomy," Journal of Clinical Oncology, vol. 23, no. 13, pp. 2911-2917, 2005.

[32] T. Y. Chan, A. W. Partin, P. C. Walsh, and J. I. Epstein, "Prognostic significance of Gleason score 3+4 versus Gleason score $4+3$ tumor at radical prostatectomy," Urology, vol. 56, no. 5, pp. 823-827, 2000.

[33] B. A. O’Brien, R. J. Cohen, T. M. Wheeler, and R. E. Moorin, "A post-radical-prostatectomy nomogram incorporating new pathological variables and interaction terms for improved prognosis," BJU International, vol. 107, no. 3, pp. 389-395, 2011.

[34] E. Kikuchi, P. T. Scardino, T. M. Wheeler et al., "Is tumor volume an independent prognostic factor in clinically localized prostate cancer?" Journal of Urology, vol. 173, no. 4, p. 1433, 2005.

[35] M. Ohori, T. M. Wheeler, M. W. Kattan, Y. Goto, and P. T. Scardino, "Prognostic significance of positive surgical margins in radical prostatectomy specimens," Journal of Urology, vol. 154, no. 5, pp. 1818-1824, 1995.

[36] R. B. Watson, F. Civantos, and M. S. Soloway, "Positive surgical margins with radical prostatectomy: detailed pathological analysis and prognosis," Urology, vol. 48, no. 1, pp. 80-90, 1996.

[37] M. Ates, D. Teber, A. S. Gözen et al., "Do tumor volume, tumor volume ratio, type of nerve sparing and surgical experience affect prostate specific antigen recurrence after laparoscopic radical prostatectomy? A matched pair analysis," Journal of Urology, vol. 177, no. 5, pp. 1771-1776, 2007.

[38] T. A. Stamey, J. E. McNeal, C. M. Yemoto, B. M. Sigal, and I. M. Johnstone, "Biological determinants of cancer progression in men with prostate cancer," Journal of the American Medical Association, vol. 281, no. 15, pp. 1395-1400, 1999.

[39] B. A. Nelson, S. B. Shappell, S. S. Chang et al., "Tumour volume is an independent predictor of prostate-specific antigen recurrence in patients undergoing radical prostatectomy for clinically localized prostate cancer," BJU International, vol. 97, no. 6, pp. 1169-1172, 2006.

[40] H. Fukuhara, H. Kume, M. Suzuki et al., "Maximum tumor diameter: a simple independent predictor for biochemical recurrence after radical prostatectomy," Prostate Cancer and Prostatic Diseases, vol. 13, no. 3, pp. 244-247, 2010.

[41] B. I. Chung, T. V. Tarin, M. Ferrari, and J. D. Brooks, "Comparison of prostate cancer tumor volume and percent cancer in prediction of biochemical recurrence and cancer specific survival," Urologic Oncology: Seminars and Original Investigations. In press.

[42] M. L. Blute, E. J. Bergstralh, A. Iocca, B. Scherer, and H. Zincke, "Use of Gleason score, prostate specific antigen, seminal vesicle and margin status to predict biochemical failure after radical prostatectomy," Journal of Urology, vol. 165, no. 1, pp. 119-125, 2001.

[43] K. Hashimoto, N. Masumori, F. Takei et al., "Prognostic value of surgical margin status for biochemical recurrence following radical prostatectomy," Japanese Journal of Clinical Oncology, vol. 38, no. 1, pp. 31-35, 2008.

[44] T. Sæther, L. T. Sørlien, T. Viset, S. Lydersen, and A. Angelsen, "Are positive surgical margins in radical prostatectomy specimens an independent prognostic marker?" Scandinavian Journal of Urology and Nephrology, vol. 42, no. 6, pp. 514-521, 2008.

[45] A. M. Lake, C. He, and D. P. Wood Jr., "Focal positive surgical margins decrease disease-free survival after radical prostatectomy even in organ-confined disease," Urology, vol. 76, no. 5, pp. 1212-1216, 2010.

[46] A. Ochiai, T. Sotelo, P. Troncoso, V. Bhadkamkar, and R. J. Babaian, "Natural history of biochemical progression after radical prostatectomy based on length of a positive surgical margin," Urology, vol. 71, no. 2, pp. 308-312, 2008.

[47] L. Masieri, M. Lanciotti, G. Nesi et al., "Prognostic role of perineural invasion in 239 consecutive patients with pathologically organ-confined prostate cancer," Urologia Internationalis. In press.

[48] A. D. Merrilees, P. B. Bethwaite, G. L. Russell, R. G. Robinson, and B. Delahunt, "Parameters of perineural invasion in radical prostatectomy specimens lack prognostic significance," Modern Pathology, vol. 21, no. 9, pp. 1095-1100, 2008.

[49] H. Miyake, I. Sakai, K. I. Harada, H. Eto, and I. Hara, "Limited value of perineural invasion in radical prostatectomy specimens as a predictor of biochemical recurrence in Japanese men with clinically localized prostate cancer," Acta Urologica Japonica, vol. 51, no. 4, pp. 241-246, 2005.

[50] J. Endrizzi and T. Seay, "The relationship between early biochemical failure and perineural invasion in pathological T2 prostate cancer," BJU International, vol. 85, no. 6, pp. 696-698, 2000.

[51] C. J. Beard, M. H. Chen, K. Cote et al., "Perineural invasion is associated with increased relapse after external beam radiotherapy for men with low-risk prostate cancer and may be a marker for occult, high-grade cancer," International Journal of Radiation Oncology Biology Physics, vol. 58, no. 1, pp. 19-24, 2004.

[52] K. J. O'Malley, C. R. Pound, P. C. Walsh, J. I. Epstein, and A. W. Partin, "Influence of biopsy perineural invasion on long-term biochemical disease-free survival after radical prostatectomy," Urology, vol. 59, no. 1, pp. 85-90, 2002.

[53] D. Van den Ouden, R. Kranse, W. C. J. Hop, T. H. Van der Kwast, and F. H. Schröder, "Microvascular invasion in prostate cancer: prognostic significance in patients treated by radical prostatectomy for clinically localized carcinoma," Urologia Internationalis, vol. 60, no. 1, pp. 17-24, 1998.

[54] A. De La Taille, M. A. Rubin, R. Buttyan et al., "Is microvascular invasion on radical prostatectomy specimens a useful predictor of PSA recurrence for prostate cancer patients?" European Urology, vol. 38, no. 1, pp. 79-84, 2000.

[55] M. K. Ferrari, J. E. McNeal, S. M. Malhotra, and J. D. Brooks, "Vascular invasion predicts recurrence after radical prostatectomy: stratification of risk based on pathologic variables," Urology, vol. 64, no. 4, pp. 749-753, 2004.

[56] D. E. Henson, R. V.P. Hutter, and G. Farrow, "Practice protocol for the examination of specimens removed from patients with carcinoma of the prostate gland: a publication of the cancer committee, College of American Pathologists," Archives of Pathology and Laboratory Medicine, vol. 118, no. 8, pp. 779783, 1994.

[57] M. L. Quek, S. Daneshmand, S. Rodrigo et al., "Prognostic significance of neuroendocrine expression in lymph nodepositive prostate cancer," Urology, vol. 67, no. 6, pp. 12471252, 2006.

[58] M. H. Weinstein, A. W. Partin, R. W. Veltri, and J. I. Epstein, "Neuroendocrine differentiation in prostate cancer: enhanced prediction of progression after radical prostatectomy," Human Pathology, vol. 27, no. 7, pp. 683-687, 1996.

[59] M. May, M. Siegsmund, F. Hammermann, V. Loy, and S. Gunia, "Prognostic significance of proliferation activity and neuroendocrine differentiation to predict treatment failure 
after radical prostatectomy," Scandinavian Journal of Urology and Nephrology, vol. 41, no. 5, pp. 375-381, 2007.

[60] G. Ahlegren, K. Pedersen, S. Lundberg, G. Aus, J. Hugosson, and P. A. Abrahamsson, "Neuroendocrine differentiation is not prognostic of failure after radical prostatectomy but correlates with tumor volume," Urology, vol. 56, no. 6, pp. 1011-1015, 2000.

[61] K. Revelos, C. Petraki, A. Scorilas et al., "Correlation of androgen receptor status, neuroendocrine differentiation and angiogenesis with time-to-biochemical failure after radical prostatectomy in clinically localized prostate cancer," Anticancer Research, vol. 27, no. 5B, pp. 3651-3660, 2007. 


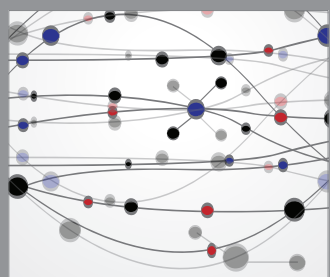

The Scientific World Journal
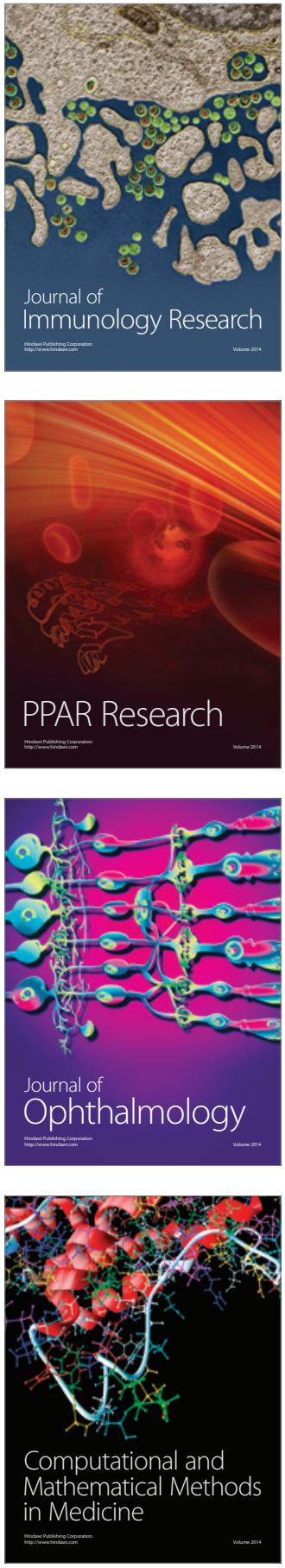

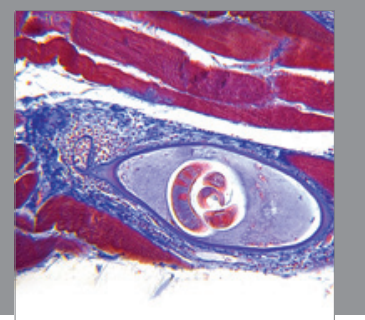

Gastroenterology

Research and Practice
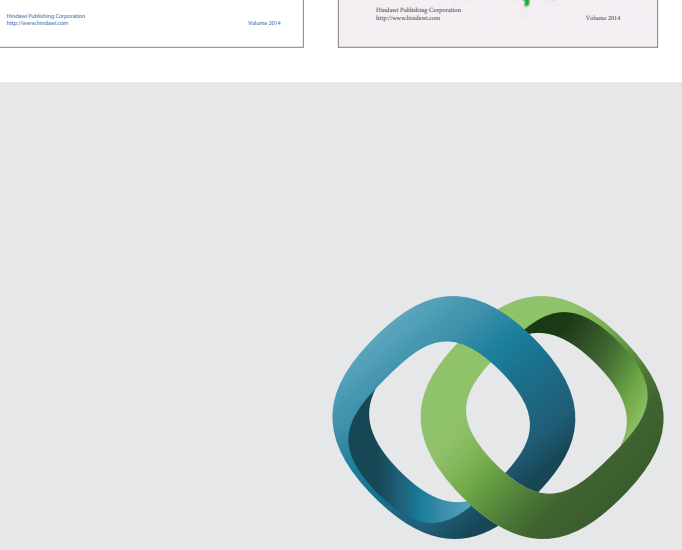

\section{Hindawi}

Submit your manuscripts at

http://www.hindawi.com
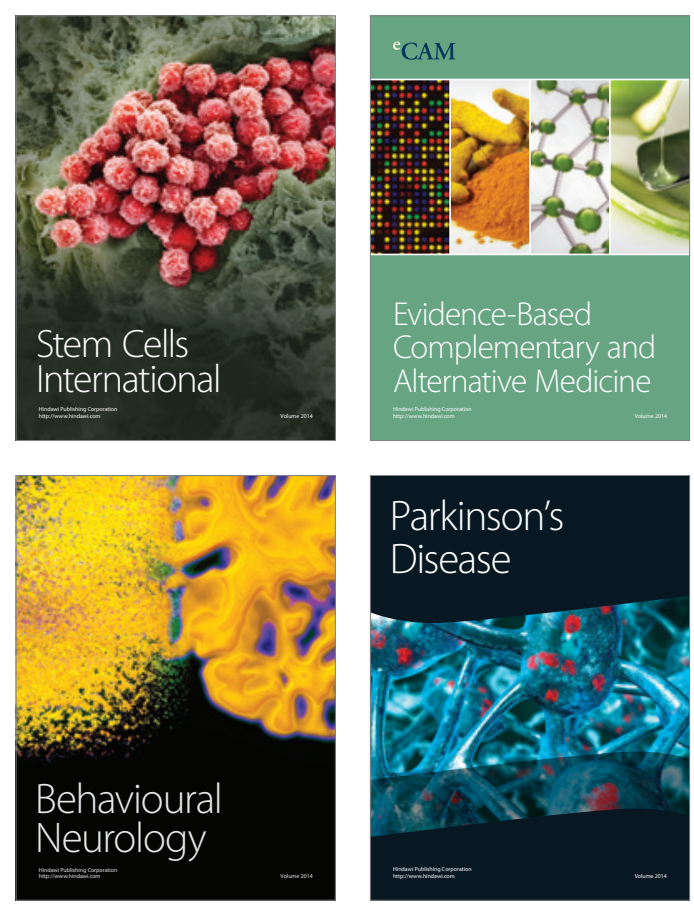

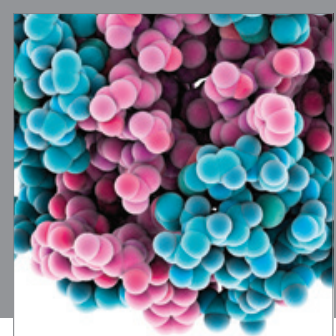

Journal of
Diabetes Research

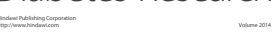

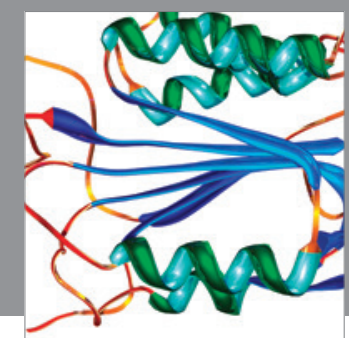

Disease Markers
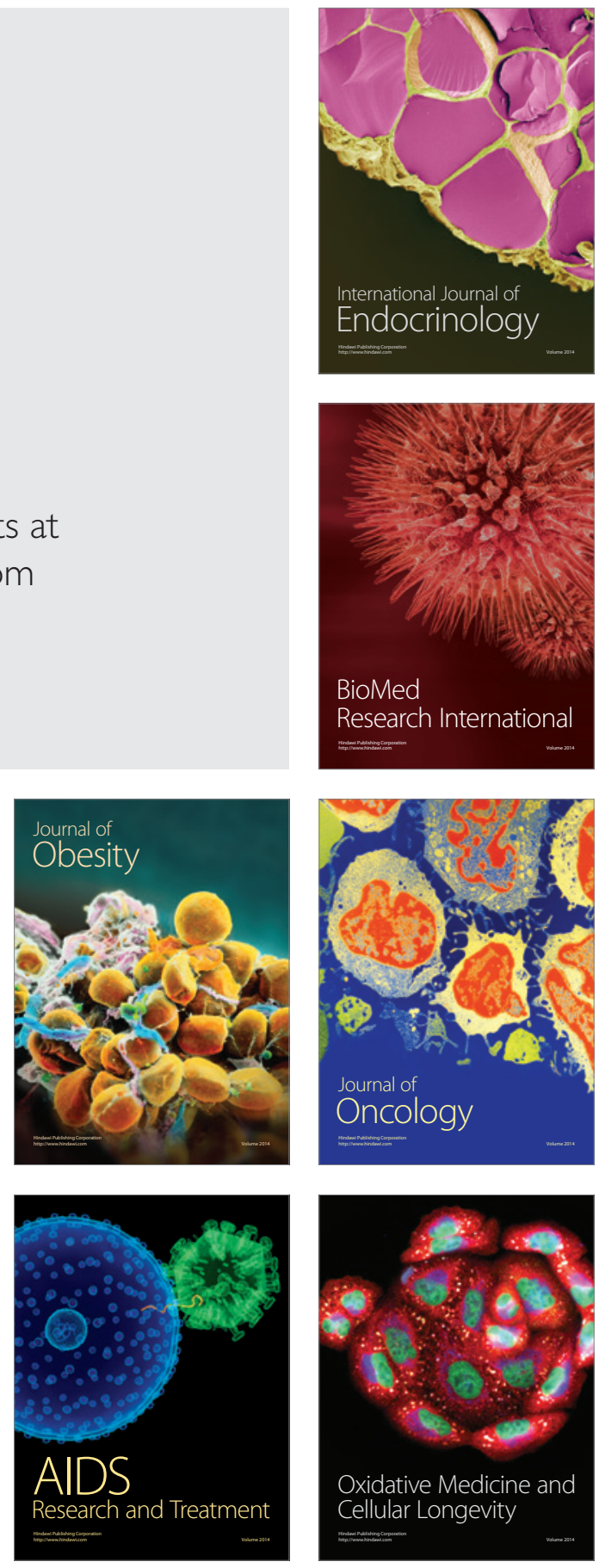